\title{
Bioinformatic analysis and prediction of the function and regulatory network of long non-coding RNAs in hepatocellular carcinoma
}

\author{
MING-RONG CAO ${ }^{1 *}$, ZE-PING HAN ${ }^{2 *}$, JI-MING LIU $^{1}$, YU-GUANG LI ${ }^{2}$, \\ YU-BING LV ${ }^{2}$, JIA-BIN ZHOU ${ }^{2}$ and JIN-HUA HE ${ }^{2}$ \\ ${ }^{1}$ Department of General Surgery, The First Affiliated Hospital, Jinan University, Guangzhou, Guangdong 510630; \\ ${ }^{2}$ Department of Laboratory, Central Hospital of Panyu, Guangzhou, Guangdong 511400, P.R. China
}

Received May 24, 2017; Accepted February 1, 2018

DOI: $10.3892 / \mathrm{ol} .2018 .8271$

\begin{abstract}
Computational analysis and bioinformatics have significantly advanced the ability of researchers to process and analyze biological data. Molecular data from human and model organisms may facilitate drug target validation and identification of biomarkers with increased predictive accuracy. The aim of the present study was to investigate the function of long non-coding RNAs (lncRNAs) in hepatocellular carcinoma (HCC) using online databases, and to predict their regulatory mechanism. HCC-associated lncRNAs, their downstream transcription factors and microRNAs (miRNAs/miRs), as well as the HCC-associated target genes, were identified using online databases. HCC-associated lncRNAs, including HOX antisense intergenic RNA (HOTAIR) and metastasis-associated lung adenocarcinoma transcript 1 (MALAT1) were selected based on established databases of lncRNAs. The interaction between the HCC-associated lncRNAs and miRNAs (hsa-miR-1, hsa-miR-20a-5p) was predicted using starBase2.0. Signal transducer and activator of transcription 1, hepatocyte nuclear factor $4 \alpha$ (HNF4A), octamer-binding transcription factor 4, Nanog homeobox (NANOG), caudal type homeobox 2 (CDX2), DEAD-box helicase 5, brahma-related gene 1, MYC-associated factor X and MYC proto-oncogene, bHLH transcription factor have been identified as the transcription factors for HOTAIR and MALAT1 using ChIPBase. Additionally, CDX2, HNF4A, NANOG, ETS transcription factor, Jun proto-oncogene and
\end{abstract}

Correspondence to: Mrs Jin-Hua He, Department of Laboratory, Central Hospital of Panyu, Guangzhou, Guangdong 511400, P.R. China

E-mail: 332518579@qq.com

${ }^{*}$ Contributed equally

Key words: hepatocellular carcinoma, long non-coding RNA, bioinformatics, regulation network forkhead box protein A1 were identified as the transcription factors for hsa-miR-1 and hsa-miR-20a-5p. CDX2, HNF4A and NANOG were the transcriptional factors in common between the lncRNAs and miRNAs. Cyclin D1, E2F transcription factor 1, epithelial growth factor receptor, MYC, MET proto-oncogene, receptor tyrosine kinase and vascular endothelial growth factor A were identified as target genes for the HCC progression, two of which were also the target genes of hsa-miR-1 and hsa-miR-20a-5p using the miRwalk and OncoDN. HCC databases. Additionally, these target genes may be involved in biological functions, including the regulation of cell growth, cell cycle progression and mitosis, and in disease progression, as demonstrated using DAVID clustering analysis. The present study aimed to predict a regulatory network of 1ncRNAs in HCC progression using bioinformatics analysis.

\section{Introduction}

Hepatocellular carcinoma (HCC) is one of the most common malignancies globally and the second-leading cause of mortality in China (1). There are $~ 62$ million newly diagnosed cases of HCC annually worldwide (2). Numerous factors, including hepatitis B virus infection and alfatoxin induction, may contribute to the development of HCC. These factors may lead to abnormal gene expression, resulting in increased cancer cell proliferation and escape from immune surveillance (3-5). The majority of patients with HCC are diagnosed at advanced stages of the disease. Currently, there is no effective surgical or pharmaceutical treatment for HCC. Therefore, investigation of the molecular mechanisms underlying HCC development is necessary to develop therapeutic strategies for HCC.

With the development of sequencing technology and the in-depth studies of oncological diseases in previous years, long non-coding RNAs (lncRNAs), once considered to be the 'noise' in genomic transcription, have come to the attention of scholars (6-8). IncRNAs are non-protein-coding transcripts that are $>200$ nucleotides, which may interact with various biomolecules, including DNA, RNA and proteins, to regulate gene expression at transcriptional, post-transcriptional and epigenetic levels (9), serving 
important functions in a wide range of biological processes, including cell proliferation, survival, differentiation and chromatin remodeling $(10,11)$. To date, the encyclopedia of DNA elements project (GENCODE v26) has conservatively annotated in humans close to 16,000 lncRNA genes that give rise to $>28,000$ distinct transcripts (12). In previous years, researchers have made notable advances in the study of IncRNA and diseases, particularly in the area of tumors (13-15). It has been suggested that the abnormal expression of lncRNA may be the major inducer and accelerant of tumorigenesis (16). However, the functions of lncRNA and their underlying molecular mechanisms are not well understood, meaning that further studies are required.

Increasingly, evidence demonstrates that a number of abnormally expressed lncRNAs are closely associated with the occurrence, invasion, metastasis and recurrence of HCC, and have great potential in its prediction, diagnosis and treatment (17). The regulatory mechanism of lncRNA is complex, and its role in HCC is diverse $(18,19)$. The present study utilized bioinformatic analysis to investigate the regulatory network of HCC-associated lncRNAs and their target genes in order further understanding on the functions of lncRNA in the pathogenesis of HCC, which is also conducive to follow-up studies.

\section{Materials and methods}

Identification of HCC-associated lncRNAs. The lncRNA disease database (http://www.cuilab.cn/lncrnadisease) has identified $>200$ types of lncRNA-associated diseases and can be utilized as a bioinformatic tool to predict human lncRNA-associated diseases (20). In the present study, HCC-associated lncRNAs were screened and candidates lncRNAs were selected using the lncRNA disease database.

IncRNA-miRNA interaction analysis. The interaction between miRNAs (6-8mer sites) and lncRNAs was predicted from the crosslinking immunoprecipitation RNA sequencing (CLIP-Seq) data using the starBase platform (http://starbase.sysu.edu.cn/) (21).

Identification of HCC-associated miRNAs. HCC-associated miRNAs were mapped into the HMDD database (http://202.38.126.151/hmdd/mirna/md/) (22).

Target gene prediction. ChIPBase (http://deepbase.sysu.edu. $\mathrm{cn} / \mathrm{chipbase} /$ ) is a database for decoding the transcriptional regulation of IncRNAs and miRNAs (23). The candidate transcription factors for 1 chRAs and miRNAs were predicted using ChIPBase and analyzed using PubMed (http://www. ncbi.nlm.nih.gov/pubmed/).

Identification of the target genes for the candidate miRNAs. miRWalk (http://www.umm.uni-heidelberg. de/apps/zmf/mirwalk/) is a comprehensive atlas of microRNA-target interactions, which provides the possible miRNA binding sites within the complete sequence of a gene (24). The target genes for the candidate miRNAs were identified using the miRWalk database.

Identification of the HCC-associated target genes. OncoDB. HCC (http://oncodb.hcc.ibms.sinica.edu.tw/index.htm) is the
Table I. Hepatocellular carcinoma-associated lncRNAs obtained from the lncRNA disease database.

\begin{tabular}{|c|c|c|c|}
\hline lncRNA & $\begin{array}{l}\text { Dysfunction } \\
\text { type }\end{array}$ & Chromosome & Genbank \\
\hline \multirow[t]{5}{*}{ H19 } & Expression & 11 & NR_002196.1 \\
\hline & Expression & 11 & NR_002196.48 \\
\hline & N/A & 11 & NR_002196.17 \\
\hline & Expression & 11 & NR_002196.1 \\
\hline & Regulation & 11 & NR_002196.1 \\
\hline HEIH & Expression & 5 & NR_045680.1 \\
\hline \multirow[t]{7}{*}{ HOTAIR } & Expression & 12 & NR_047517.1 \\
\hline & Expression & 12 & NR_047517.10 \\
\hline & Expression & 12 & NR_047517.13 \\
\hline & Expression & 12 & NR_047517.3 \\
\hline & Expression & 12 & NR_047517.1 \\
\hline & Expression & 12 & NR_047517.15 \\
\hline & Regulation & 12 & NR_047517.1 \\
\hline \multirow[t]{3}{*}{ HOTTIP } & Expression & 7 & NR_037843.3 \\
\hline & Expression & 7 & NR_037843.3 \\
\hline & Expression & 7 & NR_037843.2 \\
\hline \multirow[t]{8}{*}{ HULC } & Expression & 6 & NR_004855.2 \\
\hline & Regulation & 6 & NR_004855.2 \\
\hline & Regulation & 6 & NR_004855.2 \\
\hline & Expression & 6 & NR_004855.3 \\
\hline & Expression & 6 & NR_004855.4 \\
\hline & Expression & 6 & NR_004855.6 \\
\hline & Expression & 6 & NR_004855.2 \\
\hline & Regulation & 6 & NR_004855.2 \\
\hline IGF2-AS & N/A & 11 & NR_028044.1 \\
\hline KCNQ1OT1 & Mutation & 11 & NR_002728.3 \\
\hline \multirow[t]{7}{*}{ MALAT1 } & Expression & 11 & NR_002819.2 \\
\hline & Expression & 11 & NR_002819.2 \\
\hline & Expression & 11 & NR_002819.7 \\
\hline & Expression & 11 & NR_002819.12 \\
\hline & Expression & 11 & NR_002819.17 \\
\hline & Mutation & 11 & NR_002819.5 \\
\hline & Expression & 11 & NR_002819.2 \\
\hline \multirow[t]{3}{*}{ MEG3 } & Expression & 14 & NR_002766.2 \\
\hline & Expression & 14 & NR_002766.15 \\
\hline & Regulation & 14 & NR_002766.2 \\
\hline MINA & Expression & 3 & XR_241516.2 \\
\hline MIR7-3HG & Expression & 19 & NR_027148.1 \\
\hline \multirow[t]{2}{*}{ NPTN-IT1 } & Expression & 15 & AK055007.1 \\
\hline & Expression & 15 & AK055007.1 \\
\hline
\end{tabular}

HCC, hepatocellular carcinoma; lncRNA, long non-coding RNA; $\mathrm{HEIH}$, hepatocellular carcinoma upregulated EZH2-associated long non-coding RNA HOTAIR, HOX transcript antisense RNA; HOTTIP, HOXA distal transcript antisense RNA; HULC, highly upregulated in liver cancer; IGF2-AS, insulin-like growth factor 2-antisense RNA; KCNQ1OT1, KCNQ1 overlapping transcript 1; MALAT1, metastasis-associated lung adenocarcinoma transcript 1; MEG3, maternally expressed 3; MINA, MYC induced nuclear antigen; MIR7-3HG, MIR7-3 host gene; NPTN-IT1, NPTN intronic transcript 1; N/A, not available. 

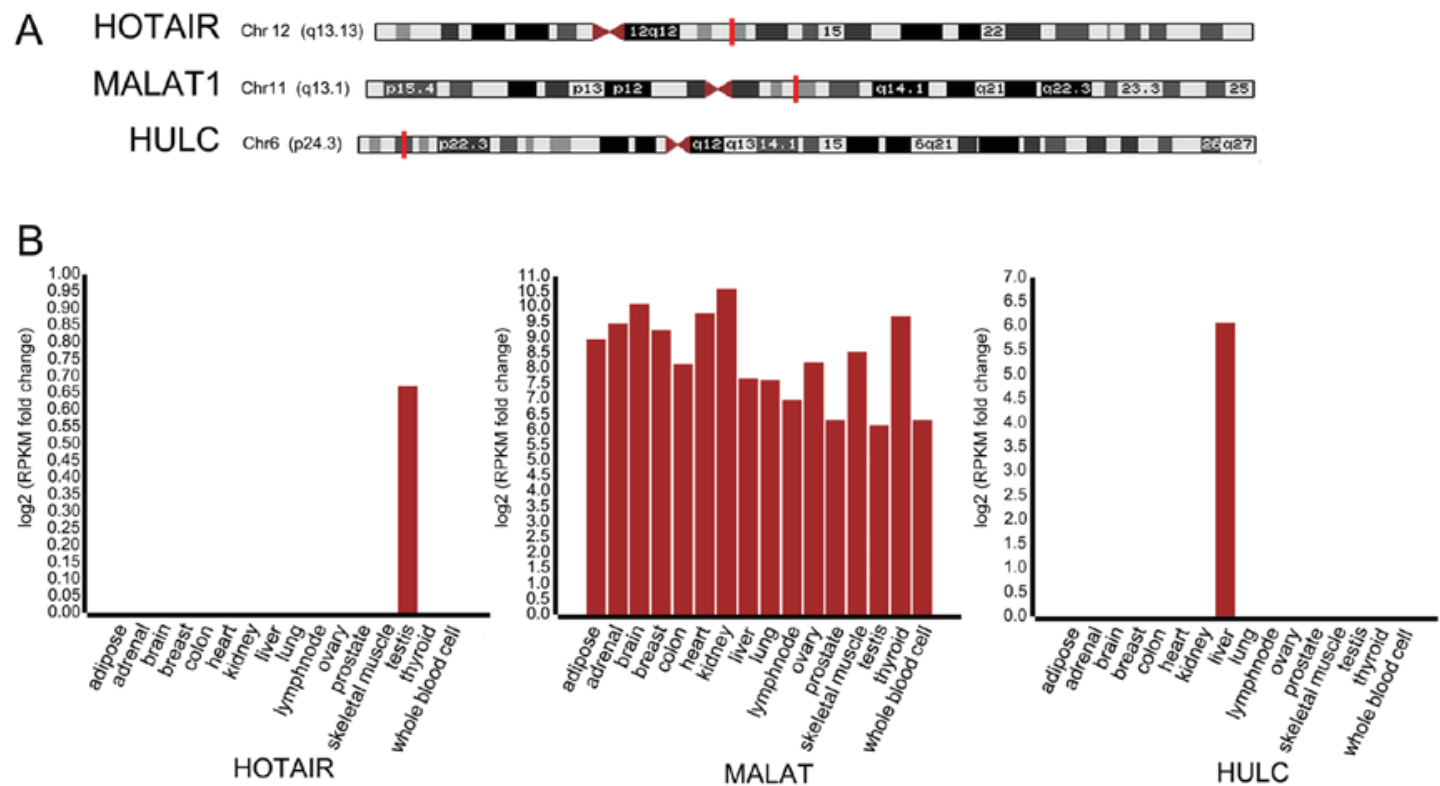

Figure 1. (A) Location of the target lncRNAs and their (B) expression pattern in different tissue. IncRNAs, long non-coding RNAs; HOTAIR, HOX antisense intergenic RNA; MALAT1, metastasis-associated lung adenocarcinoma transcript 1; Chr, chromosome; HULC, highly upregulated in liver cancer; wbc, whole blood cells.

first integrated oncogenomic database of hepatocellular carcinoma, which may aid identification aberrant cancer target genes and loci (25). Target genes that were not HCC-associated were further screened using the OncoDB.HCC database.

Functional enrichment analysis. DAVID (https://david.ncifcrf. gov/home.jsp) is a database that provides functional interpretation of a large list of genes derived from genomic studies. DAVID accelerates the analysis of genome-scale datasets by facilitating the transition from data collection to biological meaning (26). In the present study, miRNA target genes were further analyzed using DAVID, including Gene Ontology (GO) and Kyoto Encyclopedia of Genes and Genomes (KEGG) pathway analysis, and it was considered to be significant if the Benjamini-corrected P-value was $<0.05$.

Identification of transcription factors for the target lncRNAs and miRNAs. ConSite is a web-based tool for identifying cis-regulatory elements in genomic sequences (http://consite. genereg.net). Predictions are made based on the integration of binding site prediction generated with high-quality transcription factor models and cross-species comparison filtering (27). The transcription factors for each target gene were predicted using ConSite. The transcription factors that were associated with HCC were further screened using PubMed.

Construction of the regulatory network of IncRNAs in HCC. On the basis of the genomic information that was collected, the possible regulatory network of the selected lcnRNAs in HCC was constructed by Cytoscape software version 3.5.0 (http://www.cytoscape.org/index.html).

\section{Results}

HCC-associated IncRNAs. Of the 12 lncRNAs that were associated with HCC obtained from the IncRNA disease database (Table I), HOX antisense intergenic RNA (HOTAIR), metastasis-associated lung adenocarcinoma transcript 1 (MALAT1) and highly upregulated in liver cancer (HULC) were selected for further analysis.

Analyses of the target lncRNAs. The three target lncRNAs, HOTAIR, MALAT1 and HULC, were analyzed using UCSC Genome Browser. As presented in Fig. 1A, HOTAIR, MALAT1 and HULC were located on chromosome 12, 11 and 6, respectively. Additionally, MALAT1 and HULC were highly expressed in the liver, whereas HOTAIR was expressed in the liver at a lower level (Fig. 1B).

Prediction of the interaction between target lncRNAs and miRNAs. The miRNAs that were associated with HOTAIR, MALAT1 and HULC, were predicted using starBase2.0. A total of 32 miRNAs were predicted to interact with HOTAIR, and 113 miRNAs were predicted to interact with MALAT1. Among these miRNAs, miR-206, miR-1, miR-17-5p, miR-20a-5p, miR-93-5p, miR-106a-5p, miR-106b-5p, miR-613, miR-519d-5p, miR-217 and miR-20b-5p were overlapping (Fig. 2). However, no miRNA was identified to interact with HULC. Therefore, HULC was excluded from further analysis.

Identification of HCC-associated miRNAs. The HCC-associated miRNAs were obtained from HMDD and compared with the previously identified miRNAs. As presented in Fig. 3, miR-1, miR-17-5p, miR-20a-5p, miR-93-5p, miR-106a-5p, miR-106b-5p, miR-519d-5p, miR-217 and miR-20b-5p were involved in the progression of HCC and were identified to interact with HOTAIR and MALAT1. A previous study demonstrated the negative association between miRNA and lncRNA (28). Therefore, in the present study, miR-1 and miR-20a-5p were selected for further analysis, owing to their low expression in HCC. 


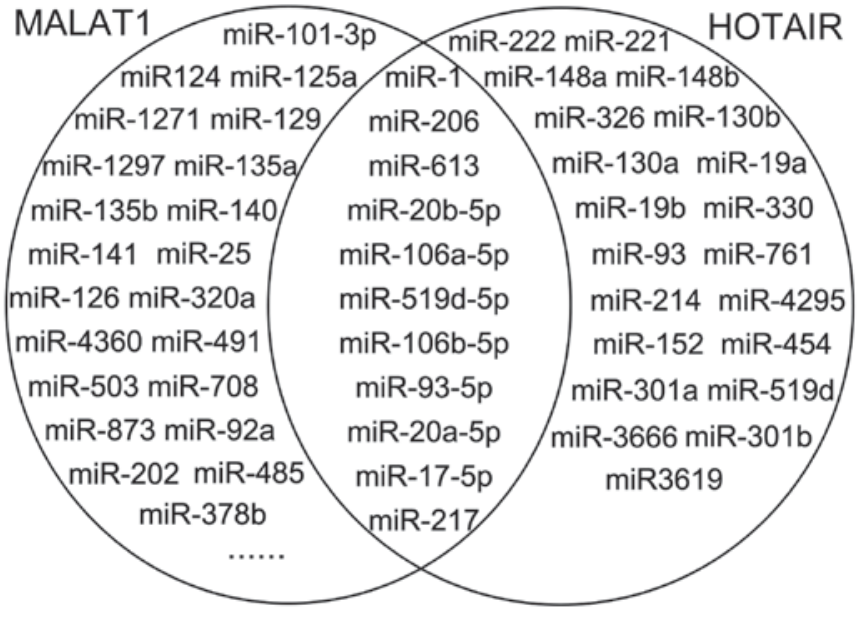

Figure 2. Prediction of interaction between MALAT1/HOTAIR and miRNAs. HOTAIR, HOX antisense intergenic RNA; MALAT1, metastasis-associated lung adenocarcinoma transcript 1 ; miRNA/miR, microRNA.

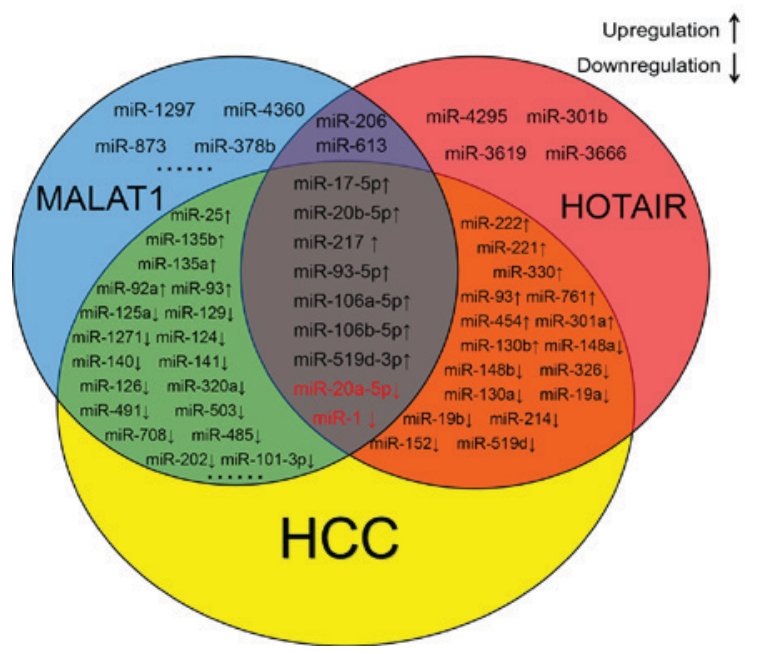

Figure 3. HCC-associated miRNAs. HCC, hepatocellular carcinoma; $\mathrm{miRNA} / \mathrm{miR}$, microRNA.

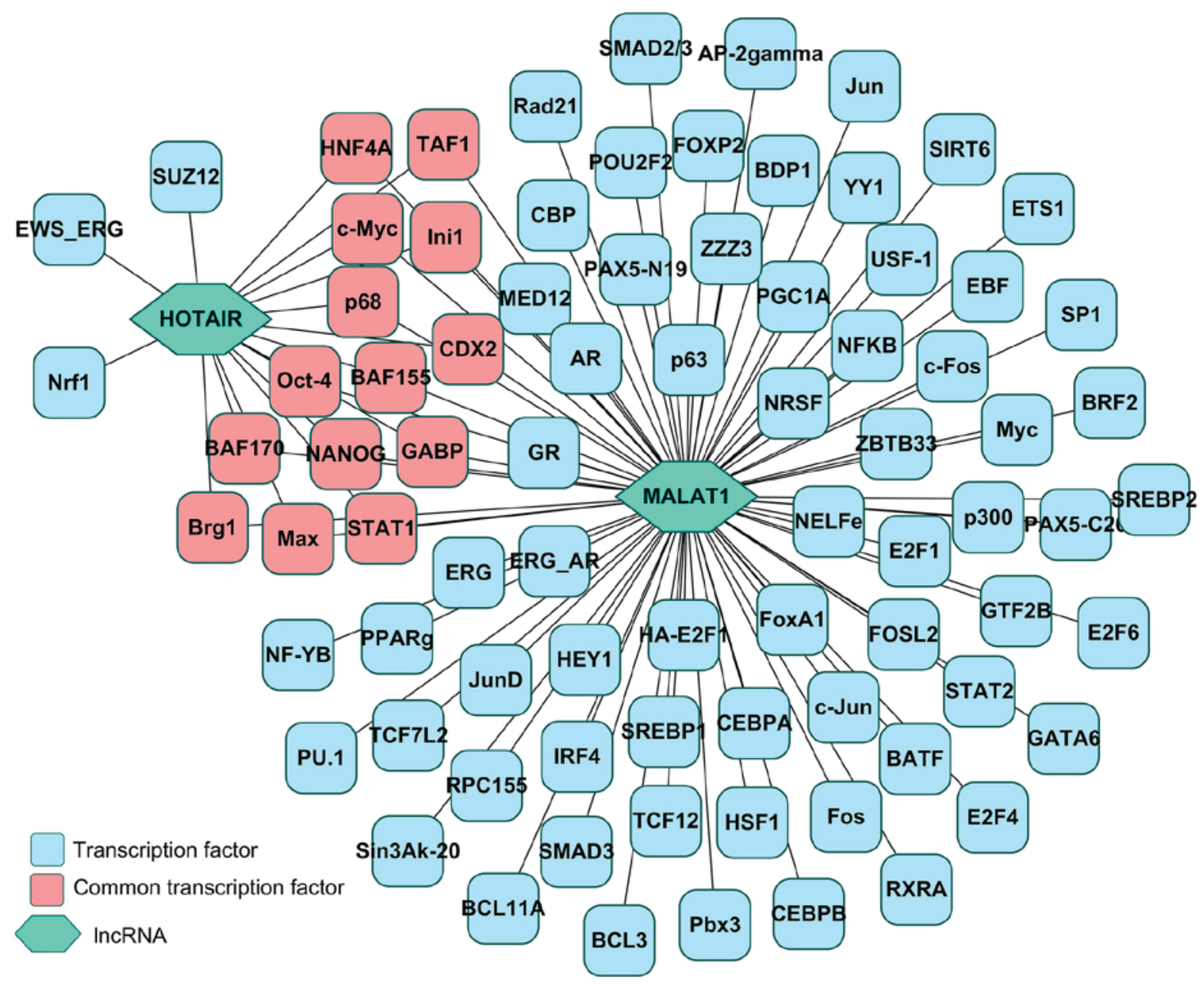

Figure 4. Prediction of transcription factors for the lncRNAs MALAT1 and HOTAIR. lncRNAs, long non-coding RNAs; HOTAIR, HOX antisense intergenic RNA; MALAT1, metastasis-associated lung adenocarcinoma transcript 1.

Prediction of the regulatory association between transcription factors and target lncRNAs/miRNAs. The transcription factors for lncRNAs (MALAT1 and HOTAIR) and miRNAs (hsa-miR-1 and hsa-miR-20a-5p) were separately predicted using ChIPBase. As presented in Fig. 4, a total of 78 and 17 transcription factors were identified for MALAT1 and HOTAIR, respectively. As presented in Fig. 5, a total of 13 and 59 transcription factors were identified for hsa-miR-1 and has-miR-20a-5p, respectively. A total of 7 overlaps were identified between hsa-miR-1 and has-miR-20a-5p (Fig. 5). 


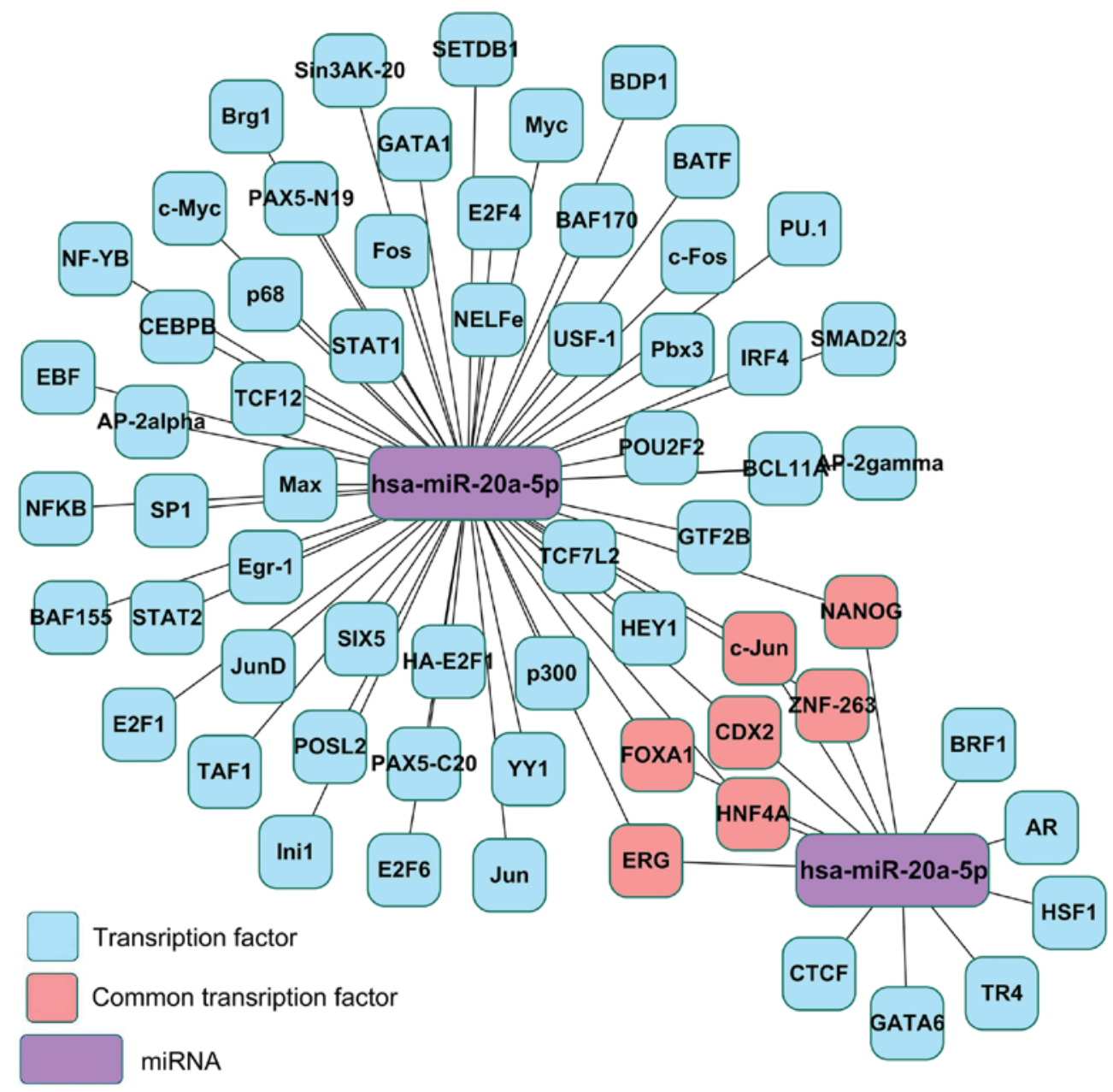

Figure 5. Prediction of transcription factors for the target miRNAs (hsa-miR-1 and hsa-miR-20a-5p). miRNA/miR, microRNA.

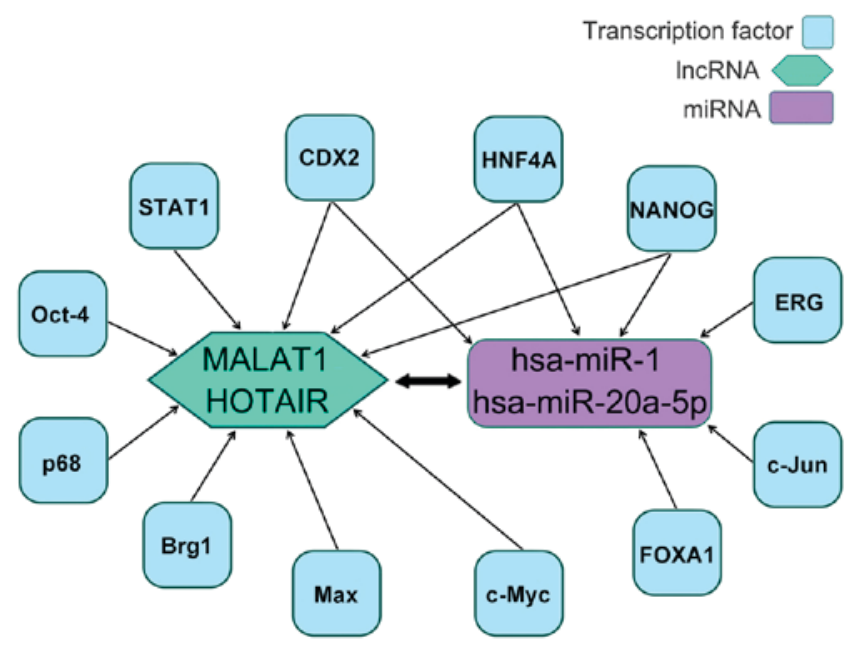

Figure 6. HCC-associated transcription factors for the target miRNAs and lncRNAs. miRNA/miR, microRNA; HCC, hepatocellular carcinoma; lncRNAs, long non-coding RNAs.

Identification of HCC-associated transcription factors. HCC-associated transcription factors were identified using PubMed. The results demonstrated that there were nine lncRNA transcription factors associated with HCC: Signal transducer and activator of transcription 1 (STAT1), hepatocyte nuclear factor $4 \alpha$ (HNF4A), octamer-binding transcription factor 4 (Oct-4), Nanog homeobox (NANOG), caudal type homeobox 2 (CDX2), DEAD-box helicase 5 (p68), brahma-related gene 1 (Brg1), MYC-associated factor X (Max) and MYC proto-oncogene, BHLH transcription factor (c-Myc) (29-36). Additionally, CDX2, erythroblast transformation-specific-related gene (ERG), forkhead box protein A1 (FOXA1), HNF4A, c-Jun and NANOG were identified as the transcription factors for hsa-miR-1 and hsa-miR-20a-5p (30-32,37-39). CDX2, HNF4A and NANOG were identified to be common between the target lncRNAs and miRNAs (Fig. 6).

Identification of target genes for hsa-miR-1 and $h s a-m i R-20 a-5 p$. The target genes for hsa-miR-1 and hsa-miR-20a-5p were predicted using the miRWalk database. A total of 879 and 101 target genes were identified for hsa-miR-1 and has-miR-20a-5p, respectively. A total of 32 overlaps were identified between these target miRNAs (Fig. 7).

Identification of the HCC-associated target genes for hsa-miR-1 and hsa-miR-20a-5p. The OncoDB.HCC database was used for the identification of the HCC-associated target genes for hsa-miR-1 and hsa-miR-20a-5p. As presented in 
Table II, among the 32 target genes, 11 of them were involved in the progression of HCC. As miRNAs are single-stranded RNA molecules that bind to targets in a base pair-mediated manner, resulting in the degradation or inhibition of the expression and function of target genes, the expression of the target genes were inverse to that of the miRNAs (40), Then, seven target genes [cyclin D1, E2F transcription factor 1 (E2F1), epithelial growth factor receptor (EGFR), MYC, MET proto-oncogene, receptor tyrosine kinase (MET), nitric oxide synthase 2A (NOS2A) and vascular endothelial growth factor (VEGF)] with increased expression in HCC were selected for the further analysis.

GO and KEGG pathway enrichment analysis of the target genes. GO and KEGG pathway enrichment analysis of identified target genes was performed using DAVID. GO analysis revealed that six genes were involved in cellular functions such as cell growth, cycle and mitosis (Table III). Additionally, KEGG analysis revealed that six genes were involved in the cell cycle and focal adhesion, and in disease progression (Table IV). NOS2A was excluded from further analysis.

Prediction of the transcription factors for the HCC-associated miRNAs. The transcription factors for the HCC-associated miRNAs were predicted using ConSite (Fig. 8). The results demonstrated that snail family transcriptional repressor 1 (Snail) was a common transcription factor for the six target genes and was involved in the progression of HCC (Table V) (41).

Functional regulatory network of lncRNA in HCC. Fig. 9 presents the proposed regulatory network of lncRNAs in HCC.

\section{Discussion}

Following advances in proteomics and genomics, the function of numerous human ncRNAs has been investigated. On the basis of their length and biological function, ncRNAs can be divided into miRNAs, small nucleolar RNA, small nuclear RNAs, piwi-interacting RNAs, natural antisense transcripts, ribosomal RNAs, transfer RNAs and lncRNAs (42). A total of 270 lncRNAs have been documented in the lncRNAdb database (20). Several studies have demonstrated that lncRNAs may serve notable functions in cell physiological activities, in the development of various types of tumor and in epigenetics, transcriptional and post-transcriptional mechanisms (42-46).

Bioinformatics is a powerful tool for genomic research that facilitates the identification of candidate genes and nucleotide sequences, and predicts the biological functions and regulatory pathways of the target genes. At present, research into lncRNAs is limited. In the present study, HCC-associated lncRNAs (HOTAIR, MALAT1 and HULC) were identified using lncRNA disease database. According to data from the UCSC Genome Browser and lncRNA disease database, HOTAIR was located on human chromosome 12 (12649 bp) and exhibited a low expression in the liver, but increased expression in HCC (47). HOTAIR may inhibit the expression of interferon- $\gamma$ and cell cycle-associated genes, thus promoting cellular invasion in HCC. Knockout of HOTAIR significantly decreased cellular proliferation and promoted apoptosis in $\operatorname{HCC}(48,49)$. The 3-year recurrence-free survival rate for patients with $\mathrm{HCC}$ that exhibited low expression of HOTAIR was significant increased compared

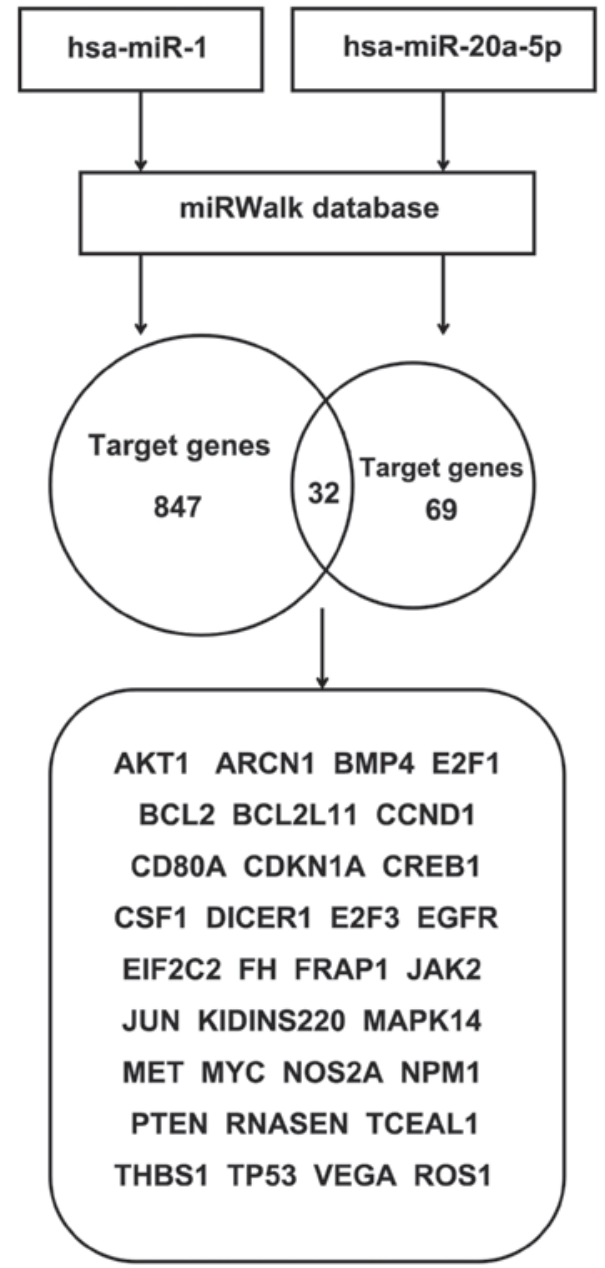

Figure 7. Shared target genes between hsa-miR-1 and hsa-miR-20a-5p obtained from the miRWalk database. miR, microRNA.

with that in patients with high expression of lncRNA following liver transplantation. Therefore, HOTAIR may be used as an independent indicator for tumor recurrence following liver transplantation (47). The results of the present study demonstrated that MALAT1 was located on human chromosome 11q13 (8708 bp), with a length of $870 \mathrm{bp}$. MALAT1 was identified in various tissues and exhibited increased expression levels in HCC (50). One previous study (51) demonstrated that MALAT1 may promote cellular proliferation in HCC, and patients with HCC with increased expression of MALAT1 exhibited an increased risk of tumor recurrence. Therefore, MALAT1 may be used for the prediction of HCC recurrence. MALAT1 may regulate the migration of HCC cells (52). The results of the present study demonstrated that HULC was located on human chromosome 6p24.3. The expression of HULC was increased in the liver but low in HCC tissues (53).

lncRNAs may interact with miRNAs (54). The results of previous studies have indicated that the interaction of lncRNAs and miRNAs serve a notable function in the progression of cancer (55-57). In the present study, starBase2.0 was used to predict the miRNAs that were associated with lncRNAs (HOTAIR, MALAT1 and HULC). However, no miRNA was identified to interact with HULC, so further analyses were performed on HOTAIR and MALAT1 only. A total of 11 overlaps were identified between HOTAIR and MALAT1, 9 of 
Table II. HCC-associated miRNA target genes obtained from the OncoDB.HCC database.

Gene Description $\quad$ Evidence

CCND1 G1/S-specific cyclin-D1 (PRAD1 oncogene)

(BCL-1 oncogene). [Source: Uniprot/SWISSPROT; Acc: P24385]

E2F1 Transcription factor E2F1 (E2F-1) (Retinoblastoma-binding protein 3) (RBBP-3) (PRB-binding protein E2F-1) (PBR3)

(Retinoblastoma-associated protein 1) (RBAP-1).

[Source: Uniprot/SWISSPROT; Acc: Q01094]

EGFR Epidermal growth factor receptor precursor (EC 2.7.10.1)

(Receptor tyrosine-protein kinase ErbB-1). [Source: Uniprot/ SWISSPROT; Acc: P00533]

MYC Myc proto-oncogene protein (c-Myc) (Transcription factor p64). [Source: Uniprot/SWISSPROT; Acc: P01106]

NOS2A Nitric oxide synthase, inducible (EC 1.14.13.39) (NOS type II) (Inducible NO synthase) (Inducible NOS)

(iNOS) (Hepatocyte NOS) (HEP-NOS).

[Source: Uniprot/SWISSPROT; Acc: P35228]

VEGFA Vascular endothelial growth factor A precursor (VEGF-A) (Vascular permeability factor) (VPF). [Source: Uniprot/ SWISSPROT; Acc: P15692]

MET Hepatocyte growth factor receptor precursor (EC 2.7.10.1) (HGF receptor) (Scatter factor receptor) (SF receptor) (HGF/ SF receptor) (Met proto-oncogene tyrosine kinase) (c-Met). [Source: Uniprot/SWISSPROT; Acc: P08581]

CDKN1A Cyclin-dependent kinase inhibitor 1 (p21) (CDK-interacting protein 1) (Melanoma differentiation-associated protein 6) (MDA-6). [Source: Uniprot/SWISSPROT; Acc: P38936]

NPM1 Nucleophosmin (NPM) (Nucleolar phosphoprotein B23) (Numatrin) (Nucleolar protein NO38). [Source: Uniprot/ SWISSPROT; Acc: P06748]

THBS1 Thrombospondin-1 precursor. [Source: Uniprot/ SWISSPROT; Acc: P07996]

TP53 Cellular tumor antigen p53 (Tumor suppressor p53) (Phosphoprotein p53) (Antigen NY-CO-13). [Source: Uniprot/SWISSPROT; Acc: P04637]
Arrays: C.R.01a, PNAS01, MBC 02

Experiments: upregulated (RT-PCR, IHC and western blotting)

Experiments: upregulated (RT-PCR)

Experiments: upregulated (IHC)

Arrays: PNAS01, Onc. 02, MBC 02

Experiments: upregulated (RT-PCR and IHC)

Experiments: upregulated (RT-PCR)

Experiments: upregulated (Northern blotting and IHC)

Experiments: upregulated (RT-PCR and mutation)

Arrays: Gast01, Onc.02, J.H.03

Experiments: downregulated (IHC and RT-PCR)

Arrays: PNAS01, MBC02, CCR03, Prot05a, Prot05c

Experiments: downregulated (qPCR)

Experiments: other (IHC, western blotting, mutation and methylation)

HCC, hepatocellular carcinoma; miR, microRNA; RT-PCR, reverse transcription-polymerase chain reaction; IHC, immunohistochemistry; qPCR, quantified polymerase chain reaction.

which were HCC-associated miRNAs according to the HMDD database. hsa-miR-1 and hsa-miR20a-5p were identified to be downregulated in HCC and selected for further analysis.

ChIPBase was used to predict the transcription factors for lncRNAs (MALAT1 and HOTAIR) and miRNAs (hsa-miR-1 and hsa-miR-20a-5p). The results demonstrated that 9 transcription factors for lncRNAs (STAT1, HNF4A, Oct-4, NANOG, CDX2, p68, Brg1, Max and c-Myc) were associated with HCC. Additionally, CDX2, ERG, FOXA1, HNF4A, c-Jun and NANOG were identified to associate with the target miRNAs and were associated with the progression of HCC. Among them, CDX2, HNF4A and NANOG were identified as the transcription factors in common for these lncRNAs and miRNAs.

In the present study, a total of 32 target genes were identified for hsa-miR-1 and hsa-miR-20a-3p using miRWalk. Among them, 11 genes were involved in the development of HCC, according to the OncoDB.HCC database. A total of seven target genes with increased expression in $\mathrm{HCC}$ (cyclin D1, E2F1, EGFR, MYC, MET, NOS2A, VEGFA) were selected for the further analysis. Additionally, GO and KEGG enrichment analysis was performed on the seven HCC-associated miRNA target genes using DAVID. The results demonstrated that six genes were involved in various cellular functions, including cell growth, cell cycle progression and mitosis. KEGG analysis revealed that six genes were involved in the cell cycle, focal adhesion and disease progression

Snail was predicted to be the transcription factor in common among the six target genes and was involved in the progression of HCC according to data from ConSite. A previous study demonstrated that upregulation of Snail may promote the proliferation and invasion of HCC cells (58). 
Table III. GO enrichment analysis for the seven HCC-associated miRNA target genes.

\begin{tabular}{|c|c|c|c|c|c|}
\hline GO ID & Term & Genes & Percentage $^{\mathrm{a}}$ & P-value & Benjamini $^{b}$ \\
\hline GO:0008283 & Cell proliferation & E2F1, EGFR, MET, MYC, VEGFA & 83.3 & $5.20 \times 10^{-6}$ & $2.40 \times 10^{-3}$ \\
\hline GO:0051726 & Regulation of cell cycle & E2F1, CCND1, EGFR, MYC & 66.7 & $1.40 \times 10^{-4}$ & $3.20 \times 10^{-2}$ \\
\hline GO:0008284 & $\begin{array}{l}\text { Positive regulation of cell } \\
\text { proliferation }\end{array}$ & CCND1, EGFR, MYC, VEFA & 66.7 & $2.70 \times 10^{-4}$ & $4.10 \times 10^{-2}$ \\
\hline GO:0044093 & $\begin{array}{l}\text { Positive regulation of } \\
\text { molecular function }\end{array}$ & CCND1, EGFR, MET, MYC & 66.7 & $7.60 \times 10^{-4}$ & $4.20 \times 10^{-2}$ \\
\hline GO:0009891 & $\begin{array}{l}\text { Positive regulation of } \\
\text { biosynthetic process }\end{array}$ & E2F1, EGFR, MYC, VEGFA & 66.7 & $1.20 \times 10^{-3}$ & $4.30 \times 10^{-2}$ \\
\hline GO:0022402 & Cell cycle process & E2F1, CCND1, EGFR, MYC & 66.7 & $6.80 \times 10^{-4}$ & $4.40 \times 10^{-2}$ \\
\hline GO:0051325 & Interphase & E2F1, CCND1, EGFR & 50 & $6.00 \times 10^{-4}$ & $4.50 \times 10^{-2}$ \\
\hline GO:0007346 & $\begin{array}{l}\text { Regulation of mitotic cell } \\
\text { cycle }\end{array}$ & CCND1, EGFR, MYC & 50 & $1.20 \times 10^{-3}$ & $4.60 \times 10^{-2}$ \\
\hline GO:0031328 & $\begin{array}{l}\text { Positive regulation of } \\
\text { cellular biosynthetic } \\
\text { process }\end{array}$ & E2F1, EGFR, MYC, VEGFA & 66.7 & $1.20 \times 10^{-3}$ & $4.90 \times 10^{-2}$ \\
\hline
\end{tabular}

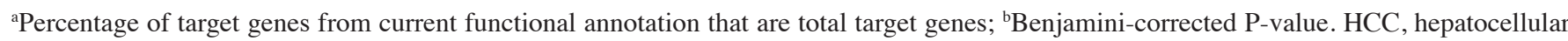
carcinoma; miR, microRNA; GO, gene ontology.

Table IV. KEGG pathway enrichment analysis for the seven HCC-associated miRNA target genes.

\begin{tabular}{|c|c|c|c|c|c|}
\hline KEGG ID & Term & Genes & Percentage $^{\mathrm{a}}$ & P-value & Benjamini ${ }^{b}$ \\
\hline hsa05219 & Bladder cancer & E2F1, CCND1, EGFR, MYC, VEGFA & 83.3 & $2.00 \times 10^{-8}$ & $7.00 \times 10^{-7}$ \\
\hline hsa05200 & Pathways in cancer & $\begin{array}{l}\text { E2F1, CCND1, EGFR, MET, MYC, } \\
\text { VEGFA }\end{array}$ & 100 & $1.10 \times 10^{-6}$ & $1.90 \times 10^{-5}$ \\
\hline hsa05218 & Melanoma & E2F1, CCND1, EGFR, MET & 66.7 & $2.60 \times 10^{-5}$ & $3.00 \times 10^{-4}$ \\
\hline hsa05212 & Pancreatic cancer & E2F1, CCND1, EGFR, VEGFA & 66.7 & $2.70 \times 10^{-5}$ & $2.30 \times 10^{-4}$ \\
\hline hsa05210 & Colorectal cancer & CCND1, EGFR, MET, MYC & 66.7 & $4.20 \times 10^{-5}$ & $3.00 \times 10^{-4}$ \\
\hline hsa04510 & Focal adhesion & CCND1, EGFR, MET, VEGFA & 66.7 & $5.70 \times 10^{-4}$ & $3.30 \times 10^{-3}$ \\
\hline hsa05213 & Endometrial cancer & CCND1, EGFR, MYC & 50 & $1.00 \times 10^{-3}$ & $5.00 \times 10^{-3}$ \\
\hline hsa05223 & Non-small cell lung cancer & E2F1, CCND1, EGFR & 50 & $1.10 \times 10^{-3}$ & $4.70 \times 10^{-3}$ \\
\hline hsa05214 & Glioma & E2F1, CCND1, EGFR & 50 & $1.50 \times 10^{-3}$ & $5.70 \times 10^{-3}$ \\
\hline hsa05220 & Chronic myeloid leukemia & E2F1, CCND1, MYC & 50 & $2.10 \times 10^{-3}$ & $7.30 \times 10^{-3}$ \\
\hline hsa05222 & Small cell lung cancer & E2F1, CCND1, MYC & 50 & $2.60 \times 10^{-3}$ & $8.30 \times 10^{-3}$ \\
\hline hsa05212 & Prostate cancer & E2F1, CCND1, EGFR & 50 & $2.90 \times 10^{-3}$ & $8.50 \times 10^{-3}$ \\
\hline hsa04110 & Cell cycle & E2F1, CCND1, MYC & 50 & $5.70 \times 10^{-3}$ & $1.50 \times 10^{-2}$ \\
\hline
\end{tabular}

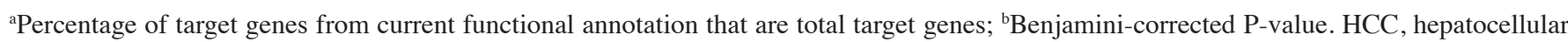
carcinoma; miR, microRNA; KEGG, Kyoto Encyclopedia of Genes and Genomes.

Therefore, in the present study a complete regulatory network of lncRNAs in HCC was proposed, whereby each one of the participants was either directly or indirectly associated and whose deregulation may result in HCC progression. Nevertheless, further research is required to confirm these regulatory associations.

In the present study the function and regulatory network of HCC-associated lncRNAs (HOTAIR and MALAT1) was predicted using bioinformatics analysis to understand the molecular mechanisms underlying the progression of HCC.

\section{Acknowledgements}

Not applicable.

\section{Funding}

The present study was supported by the First Affiliated Hospital of Jinan University (grant no. 2014106), the Technical New Star of Zhujiang, Pan Yu, Guangzhou (grant nos. 2014-special-15-3.09 and 2013-special-15-6.09), 
Table V. Prediction of the overlapping transcription factors for the six target genes using ConSite.

\begin{tabular}{|c|c|c|c|c|}
\hline Transcription factor & Sequence & From (position) & To (position) & Score \\
\hline Hunchback & TTTTTTATGC & 6,433 & 6,442 & 12.824 \\
\hline AML-1 & TTTGTGGTT & 2,631 & 2,639 & 12.626 \\
\hline c-REL & GGGGTTTTCC & 8,856 & 8,865 & 12.159 \\
\hline E74A & CCGGAAG & 6,379 & 6,385 & 10.755 \\
\hline Snail & CAGGTG & 2,151 & 2,156 & 10.744 \\
\hline Thing1-E47 & GGTCTGGCTT & 1,529 & 1,538 & 12.164 \\
\hline
\end{tabular}

TF, transcription factor; AML-1, acute myeloid leukemia protein-1; c-REL, proto-oncogene c-Rel; E74A, ETS transcription factor E74A.

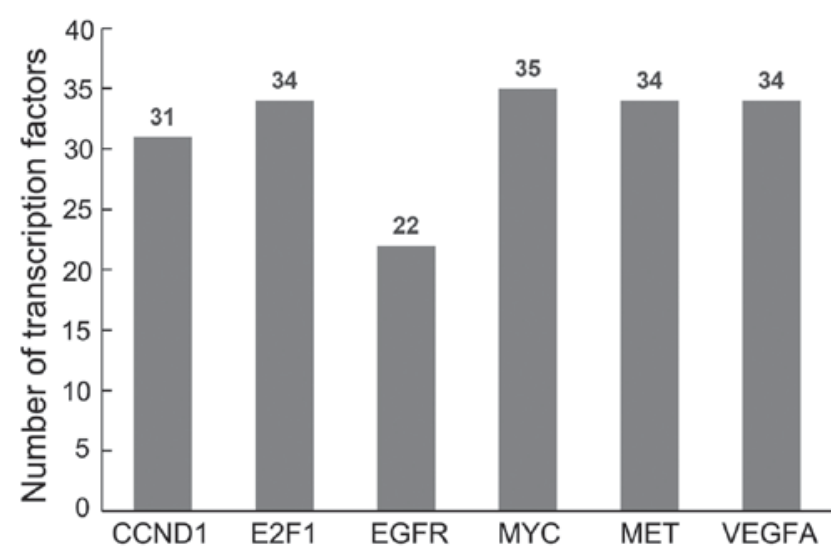

Figure 8. Prediction of the number of the transcription factors for the six target genes using ConSite. CCND1, cyclin D1; E2F1, E2F transcription factor 1; EGFR, epithelial growth factor receptor; MYC, MYC proto-oncogene, bHLH transcription factor; MET, MET proto-oncogene, receptor tyrosine kinase; VEGFA, vascular endothelial growth factor A.

the National Natural Science Foundation of China (grant no. 81502557), the Science and Technology Planning Project of Guangdong Province (grant no. 2017ZC0372) and the Medical and Health Science and Technology Project of Panyu District, Guangzhou (grant no. 2017-Z04-18).

\section{Availability of data and materials}

The data that support results of the present study are available from lncRNA disease database, starBase, HMDD, ChIPBase, PubMed, miRWalk, OncoDB.HCC, DAVID and ConSite (webpages cited in-text).

\section{Authors' contributions}

MRC drafted the article. ZPH, JML and JBZ performed the experiments. MRC and $\mathrm{ZPH}$ analysed the data and interpreted the results. YBL produced the figures and tables. MRC and ZPH revised the article. JHH and YGL conceived and designed the study. All authors read and approved the final article.

\section{Ethics approval and consent to publish}

Not applicable.

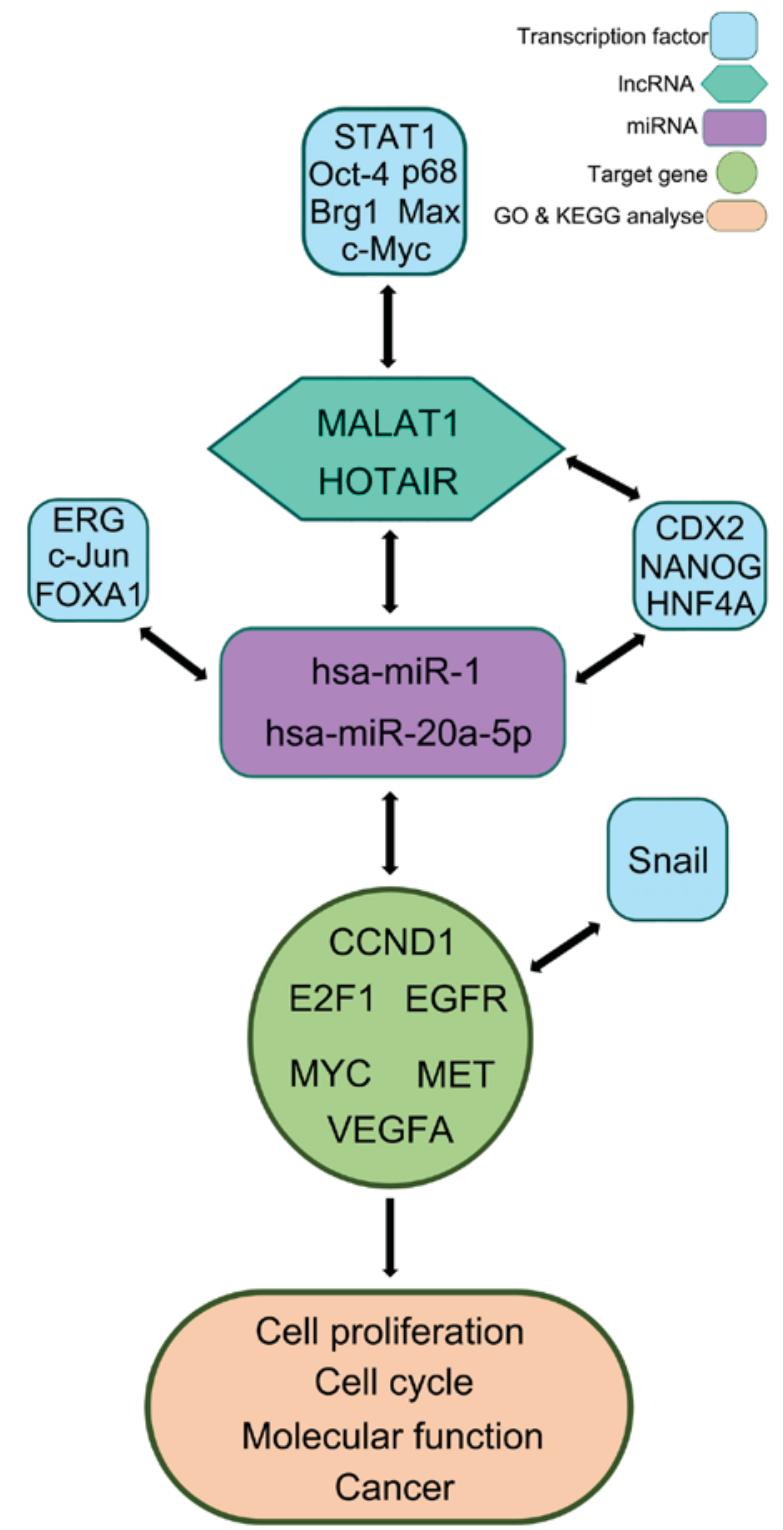

Figure 9. Proposed functional regulatory network of lncRNA in HCC. miR, microRNA; HCC, hepatocellular carcinoma; lncRNA, long non-coding RNA.

\section{Consent for publication}

Not applicable. 


\section{Competing interests}

The authors declare that they have no competing interests.

\section{References}

1. JTorre LA, Bray F, Siegel RL, Ferlay J, Lortet-Tieulent J and Jemal A: Global cancer statistics, 2012. CA Cancer J Clin 65: 87-108, 2015.

2. Siegel RL, Miller KD and Jemal A: Cancer statistics, 2015. CA Cancer J Clin 65: 5-29, 2015

3. Yang X, Xie X, Xiao YF, Xie R, Hu CJ, Tang B, Li BS and Yang SM: The emergence of long non-coding RNAs in the tumorigenesis of hepatocellular carcinoma. Cancer Lett 360: 119-124, 2015.

4. Anwar SL and Lehmann U: DNA methylation, microRNAs, and their crosstalk as potential biomarkers in hepatocellularcarcinoma. World J Gastroenterol 20: 7894-7913, 2014.

5. Roderburg $\mathrm{C}$ and Luedde $\mathrm{T}$ : The role of the gut microbiome in the development and progression of liver cirrhosis and hepatocellular carcinoma. Gut Microbes 5: 441-445, 2014.

6. Wilusz JE, Sunwoo H and Spector DL: Long noncoding RNAs: Functional surprises from the RNA world. Genes Dev 23 . 1494-1504, 2009.

7. Chew CL, Conos SA, Unal B and Tergaonkar V: Noncoding RNAs: Master regulators of inflammatory signaling. Trends Mol Med 24: 66-84, 2018.

8. Weidle UH, Birzele F, Kollmorgen G and Rüger R: Long Non-coding RNAs and their Role in Metastasis. Cancer Genomics Proteomics 14: 143-160, 2017.

9. Bassett AR, Akhtar A, Barlow DP, Bird AP, Brockdorff N, Duboule D, Ephrussi A, Ferguson-Smith AC, Gingeras TR, Haerty W, et al: Considerations when investigating lncRNA function in vivo. Elife 3: e03058, 2014.

10. Quinn JJ and Chang HY: Unique features of long non-coding RNA biogenesis and function. Nat Rev Genet 17: 47-62, 2016.

11. Marchese FP, Raimondi I and Huarte M: The multidimensional mechanisms of long noncoding RNA function. Genome Biol 18: 206, 2017.

12. Huarte M: The emerging role of lncRNAs in cancer. Nat Med 21 $1253-1261,2015$.

13. Zheng $\mathrm{P}$, Yin $\mathrm{Z}, \mathrm{Wu} \mathrm{Y}, \mathrm{Xu} \mathrm{Y}$, Luo $\mathrm{Y}$ and Zhang TC: LncRNA HOTAIR promotes cell migration and invasion by regulating MKL1 via inhibition miR206 expression in HeLa cells. Cell Commun Signal 16: 5, 2018.

14. Gutschner T, Richtig G, Haemmerle $\mathrm{M}$ and Pichler M: From biomarkers to therapeutic targets-the promises and perils of long non-coding RNAs in cancer. Cancer Metastasis Rev 37: 83-105, 2018.

15. YiRen H, YingCong Y, Sunwu Y, Keqin L, Xiaochun T, Senrui C, Ende C, XiZhou L and Yanfan C: Long noncoding RNA MALAT1 regulates autophagy associated chemoresistance via miR-23b-3p sequestration in gastric cancer. Mol Cancer 16: 174, 2017.

16. Qiu L, Tang Q, Li G and Chen K: Long non-coding RNAs as biomarkers and therapeutic targets: Recent insights into hepatocellular carcinoma. Life Sci 191: 273-282, 2017.

17. Liu YR, Tang RX, Huang WT, Ren FH, He RQ, Yang LH, Luo DZ, Dang YW and Chen G: Long noncoding RNAs in hepatocellular carcinoma: Novel insights into their mechanism. World J Hepatol 7: 2781-2791, 2015.

18. He JH, Han ZP, Liu JM, Zhou JB, Zou MX, Lv YB, Li YG and Cao MR: Overexpression of long non-coding RNA MEG3 inhibits proliferation of hepatocellular carcinoma Huh7 cells via negative modulation of miRNA-664. J Cell Biochem 118: 3713-3721, 2017

19. Malakar P, Shilo A, Mogilevsky A, Stein I, Pikarsky E, Nevo Y, Benyamini H, Elgavish S, Zong X, Prasanth KV and Karni R: Long Noncoding RNA MALAT1 promotes hepatocellular carcinoma development by SRSF1 upregulation and mTOR activation. Cancer Res 77: 1155-1167, 2017.

20. Quek XC, Thomson DW, Maag JL, Bartonicek N, Signal B, Clark MB, Gloss BS and Dinger ME: IncRNAdb v2.0: Expanding the reference database for functional long noncoding RNAs. Nucleic Acids Res 43 (Database Issue): D168-D173, 2015

21. Li JH, Liu S, Zhou H, Qu LH and Yang JH: starBase v2.0: Decoding miRNA-ceRNA, miRNA-ncRNA and protein-RNA interaction networks from large-scale CLIP-Seq data. Nucleic Acids Res 42 (Database Issue): D92-D97, 2014
22. Li Y, Qiu C, Tu J, Geng B, Yang J, Jiang T and Cui Q: HMDD v2.0: A database for experimentally supported human microRNA and disease associations. Nucleic Acids Res 42 (Database Issue): D1070-D1074, 2014.

23. Yang JH, Li JH, Jiang S, Zhou H and Qu LH: ChIPBase: A database for decoding the transcriptional regulation of long non-coding RNA and microRNA genes from ChIP-Seq data. Nucleic Acids Res 41 (Database Issue): D177-D187, 2013.

24. Dweep H, Gretz N and Sticht C: miRWalk database for miRNA-target interactions. Methods Mol Biol 1182: 289-305, 2014.

25. Su WH, Chao CC, Yeh SH, Chen DS, Chen PJ and Jou YS: OncoDB. HCC: An integrated oncogenomic database of hepatocellular carcinoma revealed aberrant cancer target genes and loci. Nucleic Acids Res 35 (Database Issue): D727-D731, 2007.

26. Huang da W, Sherman BT and Lempicki RA: Systematic and integrative analysis of large gene lists using DAVID bioinformatics resources. Nat Protoc 4: 44-57, 2009.

27. Sandelin A, Wasserman WW and Lenhard B: ConSite: Web-based prediction of regulatory elements using cross-species comparison. Nucleic Acids Res 32 (Web Server Issue): W249-W252, 2004.

28. Lin Z, Hu Y, Lai S, Xue M, Lin J, Qian Y, Zhuo W, Chen S, Si J and Wang L: Long Noncoding RNA: Its partners and their roles in cancer. Neoplasma 62: 846-854, 2015.

29. Chen G, Wang H, Xie S, Ma J and Wang G: STAT1 negatively regulates hepatocellular carcinoma cell proliferation. Oncol Rep 29: 2303-2310, 2013.

30. Yao D, Peng S and Dai C: The role of hepatocyte nuclear factor 4alpha in metastatic tumor formation of hepatocellular carcinoma and its close relationship with the mesenchymal-epithelial transition markers. BMC Cancer 13: 432, 2013.

31. Chang TS, Wu YC, Chi CC, Su WC, Chang PJ, Lee KF, Tung TH, Wang J, Liu JJ, Tung SY, et al: Activation of IL6/IGFIR confers poor prognosis of HBV-related hepatocellular carcinoma through induction of OCT4/NANOG expression. Clin Cancer Res 21: 201-210, 2015.

32. Zhu R, Wong KF, Lee NP, Lee KF and Luk JM: HNF1 $\alpha$ and CDX2 transcriptional factors bind to cadherin-17 (CDH17) gene promoter and modulate its expression in hepatocellular carcinoma. J Cell Biochem 111: 618-626, 2010.

33. Kitagawa N, Ojima H, Shirakihara T, Shimizu H, Kokubu A, Urushidate T, Totoki Y, Kosuge T, Miyagawa S and Shibata T: Downregulation of the microRNA biogenesis components and its association with poor prognosis inhepatocellular carcinoma. Cancer Sci 104: 543-551,2013.

34. Endo M, Yasui K, Zen Y, Gen Y, Zen K, Tsuji K, Dohi O, Mitsuyoshi H, Tanaka S, Taniwaki M, et al: Alterations of the SWI/SNF chromatin remodelling subunit-BRG1 and BRM in hepatocellular carcinoma. Liver Int 33: 105-117, 2013.

35. Mauleon I, Lombard MN, Muñoz-Alonso MJ, Cañelles M and Leon J: Kinetics of myc-max-mad gene expression during hepatocyte proliferation in vivo: Differential regulation of mad family and stress-mediated induction of c-myc. Mol Carcinog 39: 85-90, 2004.

36. Zhang D, Qi J, Liu R, Dai B, Ma W, Zhan Y and Zhang Y: c-Myc plays a key role in TADs-induced apoptosis and cell cycle arrest in human hepatocellular carcinoma cells. Am J Cancer Res 5: 1076-1088, 2015.

37. Hou X, Peng JX, Hao XY, Cai JP, Liang LJ, Zhai JM, Zhang KS Lai JM and Yin XY: DNA methylation profiling identifies EYA4 gene as a prognostic molecular marker in hepatocellular carcinoma. Ann Surg Oncol 21: 3891-3899, 2014.

38. Tu H, Wei G, Cai Q, Chen X, Sun Z, Cheng C, Zhang L, Feng Y, Zhou H, Zhou B and Zeng T: MicroRNA-212 inhibits hepatocellular carcinoma cell proliferation and induces apoptosis by targeting FOXA1. Onco Targets Ther 8: 2227-2235, 2015.

39. Abdou AG, Abd-Elwahed M, Badr M, Helmy M, Soliman EA and Maher D: The differential immunohistochemical expression of p53, c-Jun, c-Myc, and p21 between HCV-related hepatocellular carcinoma with and without cirrhosis. Appl Immunohistochem Mol Morphol 24: 75-87, 2016.

40. Du T and Zamore PD: Beginning to understand microRNA function. Cell Res 17: 661, 2007.

41. Zhou ZJ, Dai Z, Zhou SL, Hu ZQ, Chen Q, Zhao YM, Shi YH, Gao Q, Wu WZ, Qiu SJ, Zhou J and Fan J: HNRNPAB induces epithelial-mesenchymal transition and promotes metastasis of hepatocellularcarcinoma by transcriptionally activating SNAIL. Cancer Res 74: 2750-2762, 2014.

42. Struhl K: Transcriptional noise and the fidelity of initiation by RNA polymerase II. Nat Struct Mol Biol 14: 103-105, 2007. 
43. Caley DP, Pink RC, Trujillano D and Carter DR: Long noncoding RNAs, chromatin, and development. ScientificWorldJournal 10: 90-102, 2010.

44. Gupta RA, Shah N, Wang KC, Kim J, Horlings HM, Wong DJ, Tsai MC, Hung T, Argani P, Rinn JL, et al: Long non-coding RNA HOTAIR reprograms chromatin state to promote cancer metastasis. Nature 464: 1071-1076, 2010.

45. Vizoso M and Esteller M: The activatory long non-coding RNA DBE-T reveals the epigenetic etiology of facioscapulohumeral muscular dystrophy. Cell Res 22: 1413-1415, 2012.

46. Ishigaki S, Masuda A, Fujioka Y, Iguchi Y, Katsuno M, Shibata A, Urano F, Sobue G and Ohno K: Position-dependent FUS-RNA interactions regulate alternative splicing events and transcriptions. Sci Rep 2: 529, 2012.

47. Yang Z, Zhou L, Wu LM, Lai MC, Xie HY, Zhang F and Zheng SS: Overexpression of long non-coding RNA HOTAIR predicts tumor recurrence in hepatocellular carcinoma patients following liver transplantation. Ann Surg Oncol 18: 1243-1250, 2011.

48. Geng YJ, Xie SL, Li Q, Ma J and Wang GY: Large intervening non-coding RNA HOTAIR is associated with hepatocellular carcinoma progression. J Int Med Res 39: 2119-2128, 2011.

49. Niinuma T, Suzuki $H$, Nojima $M$, Nosho $K$, Yamamoto $H$, Takamaru H, Yamamoto E, Maruyama R, Nobuoka T, Miyazaki Y, et al: Upregulation of miR-196a and HOTAIR drive malignant character in gastrointestinal stromal tumors. Cancer Res 72: 1126-1136, 2012.

50. Tano $\mathrm{K}$ and Akimitsu N: Long non-coding RNAs in cancer progression. Front Genet 3: 219, 2012.

51. Kiss T: Small nucleolar RNA-guided post-transcriptional modification of cellular RNAs. EMBO J 20: 3617-3622, 2001.

52. Lai MC, Yang Z, Zhou L, Zhu QQ, Xie HY, Zhang F, Wu LM, Chen LM and Zheng SS: Long non-coding RNA MALAT-1 overexpression predicts tumor recurrence of hepatocellular carcinoma after liver transplantation. Med Oncol 29: 1810-1816, 2012.
53. Du Y, Kong G, You X, Zhang S, Zhang T, Gao Y, Ye L and Zhang X: Elevation of highly up-regulated in liver cancer (HULC) by hepatitis B virus X protein promotes hepatoma cell proliferation via down-regulating p18. J Biol Chem 287: 26302-26311, 2012.

54. Takeshita F, Patrawala L, Osaki M, Takahashi RU, Yamamoto Y, Kosaka N, Kawamata M, Kelnar K, Bader AG, Brown D and Ochiya T: Systemic delivery of synthetic microRNA-16 lnhibits the growth of metastatic prostate tumors via dowregulation of multiple cell-cycle genes. Mol Ther 18: 181-187, 2010.

55. Fu WM, Zhu X, Wang WM, Lu YF, Hu BG, Wang H, Liang WC Wang SS, Ko CH, Waye MM, et al: Hotair mediates hepatocarcinogenesis through suppressing miRNA-218 expression and activating P14 and P16 signaling. J Hepatol 63: 886-895, 2015.

56. Cui M, Xiao Z, Wang Y, Zheng M, Song T, Cai X, Sun B, Ye L and Zhang X: Long noncoding RNA HULC modulates abnormal lipid metabolism in hepatoma cells through an miR-9-mediated RXRA signaling pathway. Cancer Res 75: 846-857, 2015.

57. Tang Y, Jin X, Xiang Y, Chen Y, Shen CX, Zhang YC and Li YG: The lncRNA MALAT1 protects the endothelium against ox-LDL-induced dysfunction via upregulating the expression of the miR-22-3p target genes CXCR2 and AKT. FEBS Lett 589: 3189-3196, 2015.

58. Wan Z, Pan H, Liu S, Zhu J, Qi W, Fu K, Zhao T and Liang J: Downregulation of SNAIL sensitizes hepatocellular carcinoma cells to TRAIL-induced apoptosis by regulating the NF- $\kappa \mathrm{B}$ pathway. Oncol Rep 33: 1560-1566, 2015.

(1) (1) This work is licensed under a Creative Commons C. At No No Atribution-NonCommercial-NoDerivatives 4.0 International (CC BY-NC-ND 4.0) License. 